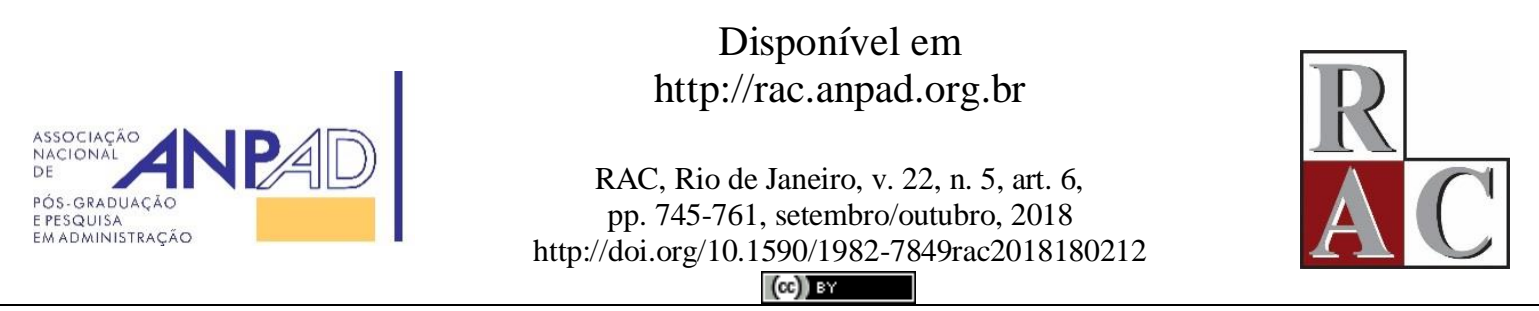

\title{
Como Escrever um Bom Caso para Ensino? Reflexões sobre o Método
}

\author{
How to Write a Good Teaching Case? Reflections on the Method
}

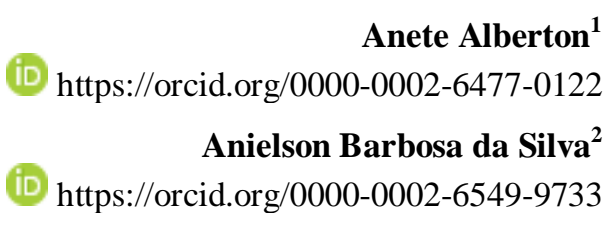

Universidade do Vale do Itajaí, Programa de Pós-Graduação em Administração, Biguaçu, SC, Brasil ${ }^{1}$ Universidade Federal da Paraíba, Departamento de Administração, João Pessoa, PB, Brasil ${ }^{2}$ 


\title{
Resumo
}

O artigo discute o método de casos para ensino no Brasil. Após a apresentação da trajetória dos casos para ensino em eventos da ANPAD, os autores refletem sobre o significado e as perspectivas teóricas que fundamentam a elaboração de casos para ensino. Discutimos os elementos que caracterizam um bom caso para ensino e os principais problemas no processo de avaliação. De forma mais específica, apresentamos diretrizes para os avaliadores de casos para ensino, enfatizando os objetivos, a relevância para a administração, as informações apresentadas e a redação do caso, além da imparcialidade do autor, do estilo do texto, da qualidade das notas de ensino e da contribuição para a área de administração. As reflexões finais retomam a questão central do título do artigo, ressaltando que a produção de um bom caso para ensino demanda o desenvolvimento de competências específicas, além de experiência e estilo de escrita autoral. Indicamos desafios para os editores de revistas e reforçamos o papel da formação de alunos de pós-graduação, professores e pesquisadores para a melhoria da qualidade na produção de casos para ensino, como também a importância da estruturação de ambientes de aprendizagem como uma estratégia inovadora para estimular a produção de casos para ensino, tanto no formato tradicional como no multimídia.

Palavras-chave: casos para ensino; aprendizagem ativa; produção de um caso para ensino.

\begin{abstract}
This article encompasses the process of writing a teaching case in Brazil. After a presentation of the trajectory of teaching cases at ANPAD conferences, the authors reflect on the meaning and the theoretical perspectives for writing a teaching case. Then, we depict the elements that characterize a good teaching case and the main problems involving the evaluation process. More specifically, we present some guidelines for reviewers of teaching cases, including the objectives, the relevance to the Management field, the information presented and the composition of the teaching case, in addition to the impartiality of the author, the text style, the quality of the teaching notes and the contribution to the management area. The final remarks argue about the central question of the title of the article, highlighting that writing a good teaching case encompasses the development of specific skills, besides having experience and a proper writing style. Therefore, we indicate some challenges for journal editors, pointing out the role of the graduate students, professors and researchers' training, to improve the quality of teaching case publication, as well as the importance of structuring learning environments as an innovative strategy to stimulate the production of teaching cases, both in traditional and in multimedia formats.
\end{abstract}

Keywords: teaching cases; active learning; writing teaching cases.

JEL codes: A20, A22, A23 


\section{Nossa Experiência com os Casos para Ensino}

Nossa relação com o tema Casos para o Ensino teve início no ano de 2001 quando os professores Anielson Barbosa da Silva e Rodrigo Bandeira-de-Mello planejaram a introdução da metodologia de avaliação e de produção de casos nas disciplinas de Tópicos Emergentes I e II do curso de Administração na Universidade do Vale do Itajaí (UNIVALI). As leituras e reflexões em torno da metodologia de casos utilizada na Universidade de Harvard e na Wharton School foram fundamentais para a delimitação de uma metodologia com aderência ao contexto do Brasil e da Univali, onde o método passou a se fazer presente na graduação e na pós-graduação lato e stricto sensu, então com a participação efetiva da Prof. ${ }^{a}$ Anete Alberton.

Posteriormente, e dada a expertise adquirida, a UNIVALI, em conjunto com outras instituições de ensino, entre elas a Universidade Federal da Paraíba (UFPB), submeteu e aprovou no Programa de Apoio ao Ensino e à Pesquisa Científica e Tecnológica em Administração (PRÓ-ADMINISTRAÇÃO - Edital 09/2008) um projeto interinstitucional intitulado Sistema de Aprendizagem-em-Ação: Metodologia de Casos no Ensino para Cursos de Graduação e Pós-Graduação em Administração. Como meta do projeto, em parceria com outras instituições brasileiras e latino-americanas, os professores autores deste artigo ministraram oficinas para professores nas Instituições de Ensino Superior (IES) parceiras, incluindo a Universidad Nacional de San Augustin em Arequipa, no Peru. Este projeto, finalizado em 2014, foi um impulsionador dos estudos sobre o método do caso, resultando em quatro dissertações de mestrado e duas teses de doutorado e a implantação das disciplinas de Casos para Ensino em Administração nos cursos de mestrado e doutorado tanto da UNIVALI como da UFPB. Estas iniciativas, além de disseminar o método, impulsionaram a formação de professores e a produção de casos, o que também refletiu-se nos encontros científicos da área.

A publicação de casos no Brasil teve um crescimento significativo no Encontro Nacional da Associação Nacional de Pós-graduação e Pesquisa em Administração (EnANPAD) a partir de 2007. Acreditamos que a publicação do artigo da professora Sylvia Roesch na Revista de Administração Contemporânea (RAC), intitulado Casos de Ensino em Administração: Notas sobre a Construção de Casos para Ensino (Roesch, 2007), também foi fundamental para o crescimento da produção de casos para ensino no Brasil e constitui um dos trabalhos de referência para professores e pesquisadores da temática no país.

Em 2009, com a mudança na estrutura dos eventos da Associação Nacional de Pós-graduação e Pesquisa em Administração (ANPAD), a partir da criação das Divisões Acadêmicas, várias ações foram empreendidas pela Divisão de Ensino e Pesquisa em Administração e Contabilidade (EPQ), visando aprimorar o processo de produção de casos para ensino. Uma dessas ações foi a reestruturação dos critérios de avaliação de casos e a elaboração de um roteiro com algumas orientações para os avaliadores, visando melhorar a qualidade do processo. Nestas iniciativas, estivemos presentes, seja na coordenação da divisão, na liderança de tema ou na participação no comitê científico da divisão EPQ até o ano de 2017.

Em 2011, a ANPAD criou a revista TAC - Tecnologias em Administração e Contabilidade, à época capitaneada pelo Prof. Rogério Quintella. O principal objetivo do periódico era valorizar e divulgar a produção caracterizada como tecnológica de programas de pós-graduação em Administração e Contabilidade e, dessa forma, realizar uma ponte entre a academia e os praticantes dessas áreas. Destaca-se que tal objetivo torna-se cada vez mais relevante, dadas à evolução acadêmica das áreas e a necessidade de engajamento profissional. Nos anos de 2015 e 2016, a Prof. a Anete Alberton assumiu, como Editora, a seção de Casos para Ensino, passando, em 2017, à Editora Associada da modalidade na Revista de Administração Contemporânea (RAC), quando da incorporação da TAC pela RAC, permanecendo na função até esta primeira metade do ano de 2018.

Neste $\operatorname{artigo~}^{(1)}$, discutimos o processo de elaboração de um bom caso para ensino. Inicialmente, apresentamos breves reflexões sobre o que é um caso para ensino, as perspectivas teóricas de aprendizagem implicadas na sua produção e análise enquanto estratégia de ensino ativa, bem como algumas especificidades e orientações que podem auxiliar alunos de pós-graduação, professores e 
pesquisadores na elaboração de um caso para ensino de qualidade na área de Administração e outras correlatas. O processo de análise e discussão de casos no contexto do ensino (Barnes, Chistensen, \& Hansen, 1994; Erskine, Leenders, \& Mauffette-Leenders, 1998; Harling \& Akridge, 1998; MauffetteLeenders, Erskine, \& Leenders, 1997) não será abordado neste artigo, mas poderá ser objeto de discussão em outras edições.

Agradecemos à editoria da RAC pelo convite e pela oportunidade de compartilhar um pouco de nossa experiência acadêmica, seja na produção, na análise e, principalmente, no ensino dos alunos de pós-graduação e futuros professores e autores de casos para ensino na área de Administração.

\section{A Trajetória dos Casos para Ensino nos Eventos da ANPAD}

A produção de casos para ensino, apesar de ter uma história de mais de cem anos em universidades americanas, foi difundida em muitas universidades brasileiras nas últimas décadas do século XX. Na década de 2000, apresentou um crescimento significativo em função de sua estruturação enquanto uma modalidade de trabalho a ser publicado nos eventos da ANPAD, visando ampliar o conhecimento sobre a análise e elaboração de casos para ensino no Brasil. Entre os anos de 2007 e 2018, os eventos da ANPAD publicaram 277 casos para ensino, cuja distribuição está representada na Figura 1.

O quantitativo é significativo, uma vez que nos últimos quatro anos foram publicados 117 casos, o que equivale a $42,24 \%$ das publicações de casos dos últimos 10 anos, indicando uma perspectiva de crescimento pela modalidade. $\mathrm{O}$ número de revistas que publicam casos para ensino também teve um incremento significativo, e já são mais de 20 revistas que os publicam regularmente. Em 2010, foi criada a Revista Brasileira de Casos para Ensino em Administração da Fundação Getúlio Vargas (FGV), a GV Casos, uma exclusividade para a modalidade, e a TAC - Tecnologias em Administração e Contabilidade pela ANPAD, especializada em artigos tecnológicos e casos para ensino, incorporada recentemente à Revista de Administração Contemporânea (RAC).

Apesar do crescimento da modalidade de casos para ensino como um produto científico e tecnológico na área de Administração, a nossa experiência como avaliadores, professores, editores e colaboradores da Divisão EPQ da ANPAD indica que muitos casos apresentam problemas em sua estruturação, tanto na delimitação do dilema como na elaboração das notas de ensino. 


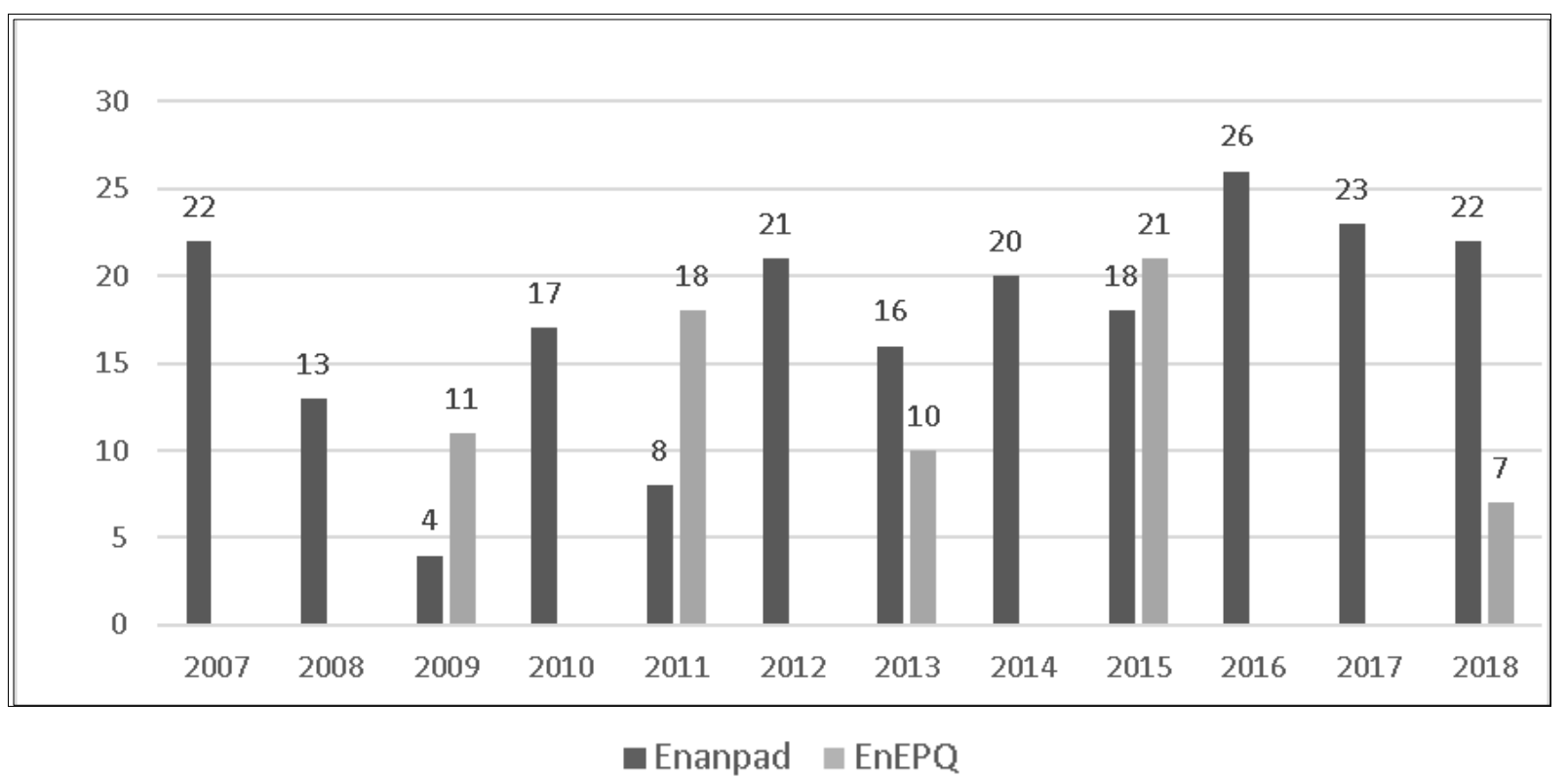

Figura 1. Casos Publicados em Eventos da ANPAD

Fonte: Elaborado pelos autores com bases nos Eventos da Associação Nacional de Pós-Graduação e Pesquisa em Administração. (2007, setembro). Encontro Nacional da Associação Nacional de Pós-Graduação e Pesquisa em Administração, Rio de Janeiro, RJ, Brasil, 31. Recuperado de http//www.anpad.org.br/ anpad/eventos.php?cod_evento=1\&cod_evento_edicao=33; Associação Nacional de PósGraduação e Pesquisa em Administração. (2008, setembro). Encontro Nacional da Associação Nacional de Pós-Graduação e Pesquisa em Administração, Rio de Janeiro, RJ, Brasil, $32 . \quad$ Recuperado de http://www.anpad.org.br/ anpad/eventos.php?cod_evento=1\&cod_evento_edicao=38; Associação Nacional de Pós-Graduação e Pesquisa em Administração. (2009, setembro). Encontro Nacional da Associação Nacional de Pós-Graduação e Pesquisa em Administração, São Paulo, SP, Brasil, 33. Recuperado de http//www.anpad.org.br/ anpad/eventos.php?cod_evento=1\&cod_evento_edicao=45; Associação Nacional de Pós-Graduação e Pesquisa em Administração. (2010, setembro). Encontro Nacional da Associação Nacional de Pós-Graduação $e$ Pesquisa em Administração, Rio de Janeiro, RJ, Brasil, $34 . \quad$ Recuperado de http:/www.anpad.org.br/ anpad/eventos.php?cod_evento=1\&cod_evento_edicao=53; Associação Nacional de Pós-Graduação e Pesquisa em Administração. (2011, setembro). Encontro Nacional da Associação Nacional de Pós-Graduação e Pesquisa em Administração, Rio de Janeiro, RJ, Brasil, 35. Recuperado de http://www.anpad.org.br/ anpad/eventos.php?cod_evento=1\&cod_evento_edicao=58; Associação Nacional de Pós-Graduação e Pesquisa em Administração. (2012, setembro). Encontro Nacional da Associação Nacional de Pós-Graduação $e$ Pesquisa em Administração, Rio de Janeiro, RJ, Brasil, $36 . \quad$ Recuperado de http:/www.anpad.org.br/ anpad/eventos.php?cod_evento=1\&cod_evento_edicao=63; Associação Nacional de Pós-Graduação e Pesquisa em Administração. (2013, setembro). Encontro Nacional da Associação Nacional de Pós-Graduação e Pesquisa em Administração, Rio de Janeiro, RJ, Brasil, 37. Recuperado de http://www.anpad.org.br/ anpad/eventos.php?cod_evento=1\&cod_evento_edicao=68; Associação Nacional de Pós-Graduação e Pesquisa em Administração. (2014, setembro). Encontro Nacional da Associação Nacional de Pós-Graduação $e$ Pesquisa em Administração, Rio de Janeiro, RJ, Brasil, 38 . Recuperado de http://www.anpad.org.br/ anpad/eventos.php?cod_evento=1\&cod_evento_edicao=73; Associação Nacional de Pós-Graduação e Pesquisa em Administração. (2015, setembro). Encontro Nacional da Associação Nacional de Pós-Graduação e Pesquisa em Administração, Belo Horizonte, MG, Brasil, 39. Recuperado de http:/www.anpad.org.br/ anpad/eventos.php?cod_evento=1\&cod_evento_edicao=78; Associação Nacional de Pós-Graduação e Pesquisa em Administração. (2016, setembro). Encontro Nacional da Associação Nacional de PósGraduação e Pesquisa em Administração, Costa do Saúpe, BA, Brasil, 40. Recuperado de http:/www.anpad.org.br/ anpad/eventos.php?cod_evento=1\&cod_evento_edicao=83; Associação Nacional de Pós-Graduação e Pesquisa em Administração. (2017, outubro). Encontro Nacional da Associação Nacional de Pós-Graduação e Pesquisa em Administração, SãoPaulo, SP, Brasil, 41. Recuperado de http://www.anpad.org.br/ anpad/eventos.php?cod_evento=1\&cod_evento_edicao=89; Associação Nacional de Pós-Graduação e Pesquisa em Administração. (2018, outubro). Encontro Nacional da Associação Nacional de Pós-Graduação e Pesquisa em Administração, Curitiba, PR, $\quad$ Brasil, $2 . \quad$ Recuperado de http:/www.anpad.org.br/ anpad/eventos.php?cod_evento=1\&cod_evento_edicao=93; Associação Nacional de Pós-Graduação e Pesquisa em Administração. (2007b, novembro). Encontro de Ensino e Pesquisa em Administração e Contabilidade, Recife, PE, Brasil, 1. Recuperado de http:/www.anpad.org.br/ anpad/eventos.php?cod_evento=18\&cod_evento_edicao=35; Associação Nacional de Pós-Graduação e Pesquisa em Administração. (2009b, novembro). Encontro de Ensino e Pesquisa em Administração e Contabilidade, Curitiba, PR, Brasil, 2. Recuperado de http//www.anpad.org.br/ anpad/eventos.php?cod_evento=18\&cod_evento_edicao=47; Associação Nacional de PósGraduação e Pesquisa em Administração. (2011b, novembro). Encontro de Ensino e Pesquisa em Administração e Contabilidade, João Pessoa, PB, Brasil, 3. Recuperado de http:/www.anpad.org.br/ anpad/eventos.php?cod_evento=18\&cod_evento_edicao=60; Associação Nacional de Pós-Graduação e Pesquisa em Administração. (2013b, novembro). Encontro de Ensino e Pesquisa em Administração e Contabilidade, Brasília, DF, Brasil, $4 . \quad$ Recuperado de http://www.anpad.org.br/ anpad/eventos.php?cod_evento=18\&cod_evento_edicao=70; Associação Nacional de Pós-Graduação e Pesquisa em Administração. (2015b, novembro). Encontro de Ensino e Pesquisa em Administração e Contabilidade, Salvador, BA, Brasil, 5. Recuperado de http//www.anpad.org.br/ anpad/eventos.php?cod_evento=18\&cod_evento_edicao=79; Associação Nacional de PósGraduação e Pesquisa em Administração. (2018b, maio). Encontro de Ensino e Pesquisa em Administração e Contabilidade, Porto Alegre, RS, Brasil, 6. Recuperado de http:/www.anpad.org.br/ anpad/eventos.php?cod_evento=18\&cod_evento_edicao=90 


\section{O Que é um CASO para ENSINO?}

No processo da difusão de casos para ensino no Brasil, muitos autores destacaram a sua diferença com o método de pesquisa de estudo de caso (Machado \& Callado, 2008; Roesch, 2007), distinção necessária não apenas em função da falta de conhecimento sobre casos no contexto do ensino, mas também porque, em inglês, os termos case study, case method (Bonoma, 1989; Ellet, 2007, 2008; Rosier, 2002), teaching case (Roberts, 1999) e teaching business cases (Rollag, 2010) são utilizados para caracterizar especificidades sobre o uso de casos no ensino. No Brasil, a ANPAD passou a adotar Casos para Ensino como uma modalidade de trabalhos e contribuiu na busca da homogeneização do termo.

Antes de discutir o significado de um caso para ensino, é relevante abordar o termo método de caso, que é amplo (Jennings, 1996, 2002) e refere-se a uma variedade de estilos de ensino e objetivos que podem ser adotados para sua utilização (Chang, 2003). O método de caso pode fomentar o envolvimento emocional e intelectual dos estudantes, contribuir para uma maior compreensão em longo prazo e dar mais realismo aos cenários da educação gerencial (Chang, 2003). Assim, quando nos referimos ao método do caso para ensino, estamos abordando um conjunto de procedimentos relacionados à elaboração ou à utilização de casos no contexto do ensino.

Um caso para ensino é uma descrição de uma situação administrativa (Bonoma, 1989), uma fonte rica de dados detalhados que representa a complexidade organizacional (Booth, Bowie, Jordan, \& Rippin, 2000), que imita ou simula uma situação real. Seu principal propósito é promover uma representação da realidade (Ellet, 2007, 2008), encorajando a tomada de decisão e o risco em um ambiente controlado. Quando utilizado no contexto do ensino, um critério determinante é que o caso deve fomentar um debate entre os estudantes (Jennings, 1996, 2002), o qual, para ser 'rico' em aprendizagem, demanda o relato de uma situação estruturada e detalhada, com um dilema bem definido. Entretanto, o processo de massificação do ensino dificulta a utilização de casos mais estruturados e muitos professores acabam aplicando casos curtos com uma ou duas páginas (Booth et al., 2000), sem coerência com a proposta dos casos para ensino hoje publicados em revistas e eventos científicos e tecnológicos na área de Administração no Brasil.

Para Ellet (2007, 2008), a caracterização de um caso deve considerar os seguintes aspectos: (a) incluir questões significativas; (b) fornecer informações suficientes para balizar as conclusões; (c) não apresentar indicação de conclusão, mas fatos que balizem o direcionamento de conclusões razoáveis. Consideramos que esses pontos indicados estão intimamente relacionados com o processo de produção de um caso. $\mathrm{O}$ autor deve construir uma descrição da situação e do dilema que possibilite ao estudante, durante o processo de análise do caso e sua posterior discussão em sala de aula, obter contribuição potencial para o desenvolvimento de competências a partir de um processo de aprendizagem experiencial e em ação, que utilize aspectos cognitivos associados ao conhecimento prévio do aluno, suas estruturas de significado, além de estimular um processo reflexivo.

A partir dessas considerações, enfatizamos que o caso para ensino é uma modalidade de trabalho que abrange um relato de uma situação vivenciada por um profissional, uma organização privada, pública ou do terceiro setor, ou ainda um projeto específico de natureza prática e/ou social, que apresenta um dilema a ser analisado no contexto do ensino de graduação ou pós-graduação. Desta forma, o método do caso subsidia o processo de tomada de decisão, oportunizando a maior integração entre a teoria e a prática. O principal objetivo é educacional e orientado para a aprendizagem e o desenvolvimento de competências específicas de uma disciplina, contribuindo para:

1. Aplicação de conhecimentos adquiridos em um curso ou disciplina.

2. Associação entre os conceitos teóricos e a prática de um determinado contexto profissional, organizacional ou institucional.

3. Desenvolvimento de um pensamento sistêmico sobre o funcionamento de uma organização ou parte dela. 
4. Compreensão da situação vivenciada por um profissional ou organização em um período de tempo determinado.

Para entender como esse processo ocorre, apresentamos a seguir algumas especificidades de perspectivas teóricas da aprendizagem que balizam a utilização de um caso para ensino nos cursos de graduação e pós-graduação em Administração.

\section{Quais as Perspectivas Teóricas que Fundamentam a Produção de um Caso para Ensino?}

Grande parte dos trabalhos sobre o método do caso não discute em profundidade as bases teóricas da aprendizagem que fundamentam a sua utilização no contexto do ensino. Como afirmou Rosier (2002), o método de caso é um processo de ensinar com casos, em que aspectos como a interação, a troca de experiências, a reflexão e a relação teoria e prática são indicados como determinantes.

Outro ponto fundamental é o potencial de contribuição da utilização de casos para ensino no desenvolvimento de competências profissionais. Como afirmaram Rocha e Mello (2000), o método pode ser utilizado como um acelerador de experiências porque expõe os alunos a várias situações em torno de um dilema que geralmente demanda uma decisão. Assim, o processo de aprendizagem do aluno deve ser mediado pela vivência de experiências e em ação, o que é um desafio para muitos professores.

Utilizar estratégias de ensino somadas às experiências em substituição aos métodos tradicionais não é um processo fácil (Herbert \& Stenfors, 2007) porque também demanda a adoção de uma orientação para aprendizagem diferente por parte dos professores. Com base em nossa experiência de ensino e de pesquisa no método do caso para ensino, enfatizamos que, para adotá-lo, é preciso conhecer os fundamentos de três perspectivas teóricas da aprendizagem: a aprendizagem construtivista, a aprendizagem experiencial e a aprendizagem em ação.

A primeira perspectiva de aprendizagem, a construtivista, abrange um conjunto de outras perspectivas que compartilham um pressuposto comum de que toda a aprendizagem está ligada à maneira como as pessoas constroem significados a partir de sua experiência. O conhecimento, na perspectiva construtivista, é um processo de construção para os aprendizes que ocorre como uma tentativa de dar sentido às suas experiências (Merriam \& Bierema, 2014). Os casos para ensino se fundamentam na aprendizagem construtivista porque os alunos ou participantes do processo de avaliação ou produção de um caso lhe atribuem significado a partir das experiências vivenciadas em todas as etapas do processo de análise e discussão no contexto da sala de aula. Dessa forma, a relação entre os professores/facilitadores e entre os alunos é um processo de negociação e troca de experiências.

A aprendizagem experiencial está no epicentro das reflexões sobre a produção e análise de casos porque se caracteriza como um processo pelo qual o conhecimento é criado por meio da transformação da experiência (D. Kolb, 1984), além de ser entendida como um conjunto de ferramentas e técnicas para estimular a vivência de experiências que promovam a aprendizagem (A. Y. Kolb \& Kolb, 2005). Ela ocorre a partir da participação ativa e responsiva do aluno no processo de aprendizagem quando há o envolvimento cognitivo, afetivo e comportamental no desenvolvimento de competências (Bangs, 2011).

A nossa experiência revela que os casos para ensino facilitam o processo de aprendizagem experiencial no ambiente da educação formal porque têm potencial de levar à transformação, como propõe Jarvis (2006), decorrente do comprometimento, análise crítica e aplicação de resultados. Silva (2014) constatou, a partir da análise de pesquisas sobre a dimensão da prática reflexiva do sistema de aprendizagem em ação, que a "relação entre as especificidades da aprendizagem experiencial e o método de caso potencializa o desenvolvimento da prática reflexiva, que ainda é pouco estudada no contexto da educação em administração" (p. 6) e constitui uma das dimensões centrais da aprendizagem experiencial (Moon, 2004). 
$\mathrm{Na}$ aprendizagem em ação, as pessoas aprendem mais eficazmente quando trabalham nos problemas em tempo real (Cho \& Egan, 2010; Revans, 2011), agindo e aprendendo como indivíduos, grupo e organização, pois refletem juntas com os colegas no seu próprio local de trabalho. Como a utilização de casos para ensino é um processo de aprendizagem experiencial que ocorre em ação, esta perspectiva teórica - a aprendizagem em ação - pode auxiliar o aprimoramento dos processos de educação gerencial e incentivar professores no contexto do ensino a adotarem estratégias de ensino que fomentem o desenvolvimento de competências profissionais a partir de um conjunto de atividades balizadas pela ação.

A aprendizagem em ação, como base para compreender o potencial de contribuição dos casos nos processos de ensino, possibilita, segundo Chang (2003): (a) o aumento da compreensão da realidade organizacional e das dimensões políticas e culturais da mudança; (b) o desenvolvimento de insights na aplicação ou falta de aplicação de conceitos e teorias para a ação; (c) a promoção de reflexão crítica relativa aos pressupostos individuais e crenças que delineiam a prática; (d) a ocorrência de melhorias nas habilidades interpessoais e o aumento da capacidade de aprender com a experiência. A aprendizagem em ação também fomenta o trabalho em grupo e encoraja os participantes a discutir, compartilhar e reunir suas ambições e experiências com o objetivo de criar algo diferente, um resultado coletivo mais efetivo (Smith, 2001), porque introduz um aprendizado por meio da ação ou durante a própria ação (Sofo, Yeo, \& Villafañe, 2010).

A reflexão sobre as bases teóricas da aprendizagem que orientam a utilização do caso para ensino enquanto estratégia ativa de aprendizagem (Silva, 2016, Silva, Lima, Sonaglio, \& Godoi, 2012) é fundamental para alunos, professores e pesquisadores compreenderem o seu potencial de contribuição para o desenvolvimento de competências dos alunos. No entanto, entendemos que um grande desafio para sua utilização em sala de aula abarca o próprio processo de produção de um caso para ensino.

\section{O que Caracteriza um Bom Caso para Ensino?}

Consideramos que escrever um caso para ensino é uma tarefa desafiadora tanto pela falta de experiência do autor, como também pela linguagem utilizada, uma vez que estamos acostumados a utilizar uma escrita mais formal e acadêmica. A competência para produzir um caso para ensino pode ser aprendida a partir de livros, como também pode ser ensinada em disciplinas específicas de casos para ensino em Administração. Como professores destas disciplinas no contexto da pós-graduação, consideramos que os resultados podem ser muito exitosos.

Algumas características de um bom caso para ensino incluem a clareza do objetivo e do dilema, a sua contribuição para a área de conhecimento da Administração e o contexto em que foi desenvolvido, bem comoa imparcialidade do autor na apresentação do caso, o fornecimento de informações detalhadas de incidentes ou diálogos abrangendo o contexto, os antecedentes, os agentes envolvidos e o dilema, além da qualidade das notas de ensino.

As orientações para a elaboração de casos para ensino foram já abordadas por vários autores (Ellet, 2007, 2008; Hawes, 2004; Ikeda, Veludo-de-Oliveira, \& Campomar, 2006; Faria \& Figueiredo, 2013; Leenders, Mauffette-Leenders, \& Erskine, 2001; Roesch, 2007; Roesch \& Fernades, 2007). Estes materiais subsidiam a construção de bons casos para ensino e sugerimos a sua leitura para aqueles que desejam se aprofundar no método.

Geralmente, um caso para ensino é estruturado em duas partes. A primeira incorpora a descrição de uma situação específica, que pode envolver um dilema associado a um problema, a uma estratégia de crescimento ou desenvolvimento, dentre outros. A segunda parte abrange as notas de ensino, que objetivam orientar o professor na análise do caso no contexto educacional, seja na graduação ou na pósgraduação. Apesar de não existir uma estrutura fixa para um caso para ensino, apresentamos a seguir alguns elementos estruturais que podem subsidiar a sua elaboração: 
1. Introdução: delimitar a época e o local em que se desenvolve o caso, bem como apresentar os protagonistas do caso e introduzir o dilema, que será detalhadamente abordado nas seções subsequentes e resgatado no fechamento do caso.

2. Contexto do caso: apresentação do contexto e dos agentes envolvidos na situação específica, vinculados ao tema abordado no caso, com a indicação de fatos objetivos abrangendo uma situação. O contexto do caso pode ser apresentado em várias seções, cujos títulos são definidos pelos autores de acordo com os objetivos e a sua criatividade.

3. Dilema do caso: após a caracterização do contexto do caso, recomenda-se que sejam apresentadas informações mais específicas para suscitar no leitor uma reflexão sobre a decisão envolvida.

4. Fechamento do caso: compreende um ou dois parágrafos finais em que o autor apresenta alguns direcionamentos sobre o dilema do caso que anunciam o seu encerramento e as decisões que precisam ser tomadas. Pode incluir alguns questionamentos mais reflexivos que delimitam claramente o escopo do caso e vão subsidiar a construção das questões indicadas nas notas de ensino.

5. Anexos e/ou Apêndices: devem ser indicados antes das notas de ensino e abrangem informações que podem subsidiar a análise do caso em sala de aula.

6. Notas de ensino: objetivam orientar o professor na resolução do caso em sala de aula e podem contemplar os seguintes itens: (a) fonte de dados: especificar as fontes específicas dos dados relatados no caso: entrevistas, relatórios das empresas, pesquisas de campo, sites, etc.; (b) objetivos didáticos ou educacionais: delinear qual(is) o(s) objetivos do caso, que temas serão trabalhados em aula/sessão de análise do caso em sala de aula, em que disciplinas/temas o caso pode ser aplicado/analisado, qual o nível dos alunos/participantes a quem o caso se destina (Graduação? Mestrado? Formação executiva?); (c) questões para discussão do, com a respectiva caso análise/discussão; (d) sugestão de um plano de ensino, delimitando claramente a metodologia de aplicação e análise em sala de aula; (e) revisão de literatura, apresentando as principais perspectivas teóricas que fundamentaram o caso e vão subsidiar a sua análise e direcionar caminhos de ação na discussão das questões. Todas as citações no corpo do texto da base teórica que fundamenta o caso devem utilizar as normas vigentes no evento ou periódico, em geral ABNT ou APA; (f) discussão (ou análise do caso): essa seção pode ser específica, mas também pode ser incorporada à medida que é exposta a teoria que subsidia a análise do caso, como indicado no item anterior; (g) referências (de acordo com as normas adotadas pelo evento ou periódico).

Na descrição dos itens de 1 a 5 do caso para ensino, não se deve fazer citações diretas ou indiretas de autores (estas devem constar nas notas de ensino no item e e f), mas deve ficar evidente a existência de uma teoria subjacente ao caso que vai subsidiar a sua resolução e o seu vínculo com conhecimentos específicos de uma ou mais disciplinas de um curso. Caso o autor incorpore informações de fontes públicas que não sejam de livros ou artigos científicos, recomenda-se indicar a fonte na medida em que forem apresentadas, no próprio texto, em notas de rodapé ou de final de texto; estas fontes devem também ser indicadas no item específico das notas de ensino.

\section{Principais Problemas na Avaliação de Casos para Ensino?}

A avaliação de um caso para ensino é uma etapa determinante para a publicação de casos com qualidade e com potencial para desenvolver competências nos estudantes de acordo com a temática abordada.

Vários eventos e periódicos acadêmicos e tecnológicos disponibilizam orientações para a submissão de casos, mas discutir os problemas de avaliação pode ser uma oportunidade de auxiliar autores a melhorar a qualidade dos casos produzidos e o sucesso de aprovação e publicação. Para tornar o processo mais didático, vamos utilizar os fatores de avaliação de um caso para ensino utilizados nos 
eventos da ANPAD e, a partir de nossa experiência como professores e avaliadores, indicar os principais problemas vinculados a cada um dos critérios.

Destacamos, no entanto, alguns aspectos mais gerais sobre um bom caso para ensino porque consideramos que eles são fundamentais para balizar o processo de avaliação. Inicialmente, o autor de um caso precisa definir de forma clara o tema, o contexto, os protagonistas, o dilema e a(s) teoria(s) que estarão subjacentes na descrição do caso e que serão evidenciadas na segunda parte, que compreende as notas de ensino. Ao redigir um caso, a metáfora da pirâmide invertida pode ser útil, iniciando com aspectos mais gerais abrangendo o contexto, sujeitos e fatos relevantes para, de forma mais específica, delimitar o dilema. Este talvez seja um dos problemas mais comuns na avalição de um caso: a falta de clareza do dilema.

Um bom dilema envolve o leitor e o leva a refletir sobre as possibilidades de resolução do caso, estimulando-o a se tornar protagonista da situação. Um dilema não abrange apenas um problema, mas pode estar relacionado a um projeto, a uma estratégia a ser implantada ou até a mudanças associadas ao comportamento individual ou coletivo, como também a aspectos culturais em nível mais amplo. $\mathrm{O}$ mais importante é que o dilema vai orientar a buscar alternativas para a análise e discussão do caso e precisa ter uma base teórica subjacente a ser explorada pelo aluno no momento de pensar alternativas para solucioná-lo, seja por meio da análise das questões para discussão, de um posicionamento sobre uma decisão a ser tomada ou até de um caminho a ser seguido, que pode ser estimulado pelo professor durante a discussão do caso em sala de aula. Na metáfora da pirâmide invertida, o dilema caracteriza a parte mais estreita e direciona o fechamento da primeira parte do caso.

De forma mais pontual, doravante buscamos responder a seguinte questão: quais os principais problemas na avaliação dos casos?

1. Objetivos do caso: o avaliador considera se o caso apresentado caracteriza de forma adequada a situação e o período, como também se está adequado para ser utilizado em uma disciplina. Além disso, o avaliador identifica se há uma teoria subjacente ao caso que norteia a sua descrição e também o dilema. Quando o autor não consegue apresentar um caso com clareza nos pontos indicados, a avaliação fica comprometida.

2. Relevância para a Administração: identificar se um caso é relevante para a administração demanda do avaliador a capacidade de perceber se o dilema está claro e bem delimitado e se tem potencial para relacionar a teoria com a prática. A falta de clareza na delimitação do dilema, assim como a não identificação de uma ou mais teorias subjacentes presentes no caso, dificultam a análise deste critério pelo avaliador e são os problemas mais comuns detectados no processo de avaliação. Ter uma teoria subjacente não significa indicar citações de autores, mas perceber, a partir dos fatos e situações narradas no caso por seus protagonistas, que existem teorias de base que fundamentam a solução do dilema proposto. Se o dilema não está claro, as teorias de base também não ficam evidenciadas para o avaliador.

3. Informações do caso: este critério de avaliação está intimamente vinculado à descrição de informações que vão auxiliar o analista a tomar decisões envolvendo o dilema apresentado. Essas informações precisam estar apresentadas de forma estruturada, cronológica e encadeada sobre o contexto, os protagonistas, a estrutura de uma organização ou comportamentos gerenciais (se for esta a situação). Para refletir sobre o dilema a partir das questões de análise indicadas nas notas de ensino ou propostas pelo professor, o analista deve ter todas as informações necessárias apresentadas no caso e esse será o ponto central que balizará a avaliação deste item.

4. Redação do caso: o processo de produção escrita de um caso é desafiador, pois demanda originalidade do autor. A linguagem deve ser objetiva, capaz de envolver o aluno (e o analista do caso) e fazê-lo se sentir motivado e implicado na leitura. O nível de linguagem mais adequado é aquele que torna o leitor o protagonista do caso. Geralmente, os principais problemas na avaliação deste critério incluem a falta de clareza, de organização e da capacidade de despertar o interesse do leitor, como também a falta de detalhes de situações ou diálogos dos protagonistas. A indicação de 
fontes, quando utilizadas, também é fundamental e, sempre que for necessário, o uso de anexos e apêndices também pode ser determinante na resolução do caso. Dessa forma, o avaliador deve analisar se há uma boa ligação entre as informações do caso, a indicação das fontes utilizadas e a sua correta apresentação em apêndices ou anexos quando forem necessários. Sobre o estilo linguístico do caso não há uma estrutura formal quanto ao aspecto discursivo. Pode-se usar, por exemplo, uma narrativa mais informal mantendo o objetivo central que é auxiliar na formação profissional, na integração teoria e prática, pois, do ponto de vista discursivo, conforme Araújo e Sousa (2016), o caso para ensino é um gênero textual.

5. Imparcialidade do autor: ser imparcial ao produzir um caso significa evitar descrever situações e fatos que expressem o juízo de valor do autor, bem como evitar o uso de expressões ou de adjetivos que acabem comprometendo esse posicionamento mais imparcial. Em muitos casos, os autores não conseguem fugir de uma linguagem acadêmica e muitos até incluem citações de autores na sua descrição, o que é outro problema recorrente a ser evitado pois implica um nível de parcialidade ao indicar um posicionamento teórico específico. É preciso lembrar que o método do caso é o indutivo. Quando sugerimos que a teoria deve estar subjacente ao caso não significa que ela seja explícita por meio de citações, mas que o leitor consiga fazer uma conexão com alguma teoria de base já conhecida para fundamentar a sua resolução. Em relação à imparcialidade, um bom caso para ensino é aquele que possibilita o surgimento de diversas interpretações pelos alunos em uma seção de análise de casos em sala de aula.

6. Estilo do texto: analisar o estilo do texto é um critério que está interligado com outros já indicados. Os principais problemas na avaliação de um caso ocorrem quando os autores não apresentam incidentes, diálogos ou eventos que possibilitem ao leitor delimitar com clareza o dilema. O estilo também é prejudicado quando o autor não consegue ser imparcial, e essa falta de imparcialidade pode acontecer quando o mesmo utiliza relatos de personagens extraídos de entrevistas por exemplo, sem a devida citação ou menção ao eles. Outros problemas no estilo do texto estão associados à dificuldade do autor em levar o leitor a se conectar com o dilema de modo que ele se sinta protagonista do caso. $\mathrm{O}$ mais importante para a boa avaliação deste critério é desenvolver um estilo original, próprio, uma vez que não existe um padrão em termos de estilo. Porém, considerar os problemas apresentados anteriormente pode auxiliar a obter uma boa avaliação neste item.

7. Qualidade das notas de ensino: nossa experiência indica que as notas de ensino apresentam os maiores problemas na avaliação de um caso e isso pode acontecer em função de vários motivos. Primeiro, pela falta de experiência docente do autor do caso, que tem dificuldade de visualizar como ele pode ser aplicado em sala de aula. Por isso, a experiência de aplicar um caso já publicado pode ser uma etapa inicial importante no contexto do ensino. Outra recomendação é definir questões do caso e, por meio de orientações de outros casos já publicados, testá-lo e, em seguida, fazer ajustes para a estruturação das notas de ensino. Geralmente, as notas de ensino devem apresentar uma descrição dos objetivos educacionais, incluindo as disciplinas a que o caso se destina, bem como das possíveis atividades/estrutura para análise e discussão do caso em sala de aula, além da indicação das questões e das possíveis alternativas para a resolução do caso, de forma articulada ou não com a literatura. Quando as alternativas não estão articuladas com a teoria de base que fundamenta e sustenta a resolução do caso, o autor deve criar uma seção específica para apresentação do referencial teórico associado às questões de análise. Por fim, deve-se apresentar as referências utilizadas nas notas de ensino, como também outras indicações bibliográficas que podem ser utilizadas pelo professor no momento da análise. Os principais problemas que comprometem a qualidade das notas de ensino incluem a falta de indicação de cursos ou disciplinas para os quais o caso é recomendado; a falta de clareza na delimitação dos objetivos educacionais; questões pouco desafiadoras e que abordam aspectos que não estão bem definidos no caso; a falta de ligação entre o contexto e a literatura ou abordagens teóricas relacionadas ao caso.

8. Contribuição para a Administração: esse critério está vinculado ao potencial do caso para promover a aprendizagem e o desenvolvimento de competências dos alunos, bem como para inovar em algo significativo na área. A avaliação desse critério geralmente é comprometida quando o caso 
apresenta problemas em itens de avaliação envolvendo a relevância e quando as informações do caso não apresentam elementos que deixem clara essa contribuição.

\section{Reflexões Finais}

A questão central do título deste artigo foi como escrever um bom caso para ensino? A resposta a esse questionamento compreende algumas reflexões.

Escrever é uma competência que demanda conhecimento, experiência e prática. Em termos de conhecimento, é preciso entender o que é um caso para ensino, como também as bases teóricas da aprendizagem que fundamentam a produção de um bom exemplar do gênero. Também é preciso ter uma competência teórica sobre o tema a ser abordado, com ênfase nos autores de base e no conhecimento prévio necessário para resolver o caso.

Em termos de experiência, escrever um bom caso para ensino implica em um processo de aprendizagem experiencial e em ação. A partir do conhecimento, o autor deve também levar as suas experiências profissionais vivenciadas ou conhecer as estratégias de coleta de dados necessárias para levantar as informações para elaboração do caso, que podem se realizar a partir de entrevistas ou de fontes públicas de informações, como documentos, relatórios etc.

A prática de escrever um caso implica na definição de um estilo único para envolver o leitor, desenvolvida a partir de um processo de estruturação do caso em duas partes: a primeira inclui a delimitação do caso até a apresentação do dilema, com fatos que remetam a convergências e divergências de ações e pontos de vista e que demandem a reflexão para a tomada de decisão, tendo como suporte alguma teoria subjacente; e a segunda contempla as notas de ensino que sirvam como norteadoras (mas não necessariamente delimitadoras) para o professor utilizar o caso em uma disciplina ou contexto específico, com objetivos educacionais claros que façam a 'ponte' da teoria com a prática.

Uma das dificuldades encontradas pelos professores que utilizam casos publicados em periódicos é que a maioria das revistas, incluindo a RAC, publica as notas de ensino com acesso livre para todos os leitores. Naturalmente, um dos desafios da editoria da revista é rever o processo de publicação de casos para ensino em sua própria plataforma, avaliando e, se necessário, criando restrições para acesso às notas de ensino. Porém, ressaltamos ser este um posicionamento por vezes controverso entre os professores e pesquisadores, e debates ainda necessitam ser empreendidos sobre o tema.

Nosso objetivo é suscitar reflexões sobre os fatores determinantes na formação de estudantes, futuros profissionais e pesquisadores, com competências para elaborar um caso para ensino e utilizá-lo de forma adequada para o desenvolvimento de competências profissionais dos alunos. Ellet $(2007,2008)$ reforça essa discussão ao destacar que a falta de preparação das pessoas, sejam professores ou alunos, para elaborar e utilizar casos no contexto do ensino pode levar o método à falência. Isso também não significa que a utilização de casos seguindo os pressupostos do método de forma cuidadosa também promova os melhores resultados, uma vez que existem vários fatores que vão influenciar no processo.

Por outro lado, consideramos que a preparação de alunos da pós-graduação e dos professores é um fator determinante para alcançar bons resultados com o uso de casos como uma estratégia inovadora de ensino. Além disso, devemos conscientizar os alunos sobre as especificidades de um caso para ensino e destacar as principais bases teóricas da aprendizagem que o fundamentam, sobretudo a necessidade de que o aluno se torne o protagonista de seu processo de aprendizagem. Como afirma Bonoma (1989, p. 9), "no método do caso, os pássaros aprendem a voar; com outras técnicas, elas geralmente recebem um ticket no aeroporto". Os pássaros são uma metáfora que representa os alunos.

A implementação de ambientes de aprendizagem utilizando casos para ensino, como uma central de casos para ensino multimídia, com uso de uma variedade de recursos pedagógicos (como vídeos, entrevistas, gráficos, mapas e outros elementos interativos) pode ser um direcionamento importante para 
promover inovações no uso da estratégia pelos alunos por meio de plataformas virtuais, com casos desenvolvidos a partir de dramatizações teatrais que ilustrariam algum dilema a ser analisado por alunos ou profissionais. As respostas poderiam ser postadas numa estrutura similar a um grupo de discussão, vinculado a uma disciplina ou curso, com possibilidade de interação entre os participantes do grupo. Também poderiam ser implementados grupos de discussão para o debate dos professores sobre possíveis notas de ensino em torno de determinado caso. Para motivar os alunos a participarem do caso, os autores poderiam apresentar vídeo, entrevistas, elementos interativos, introduzindo a temática e o dilema.

Enfim, ainda temos um longo caminho a percorrer, quer seja na análise, discussão e produção de casos no formato tradicional, quer seja na elaboração de novos formatos que privilegiem a evolução tecnológica que vivenciamos. A discussão sobre casos multimídia precisa avançar no Brasil (como já ocorre na Harvard Business School, na IE Business School, na Kellogg School of Management, entre outros centros de excelência internacional) o que certamente demanda a utilização de formatos diferenciados e a necessidade de definir uma estrutura específica de submissão, publicação e utilização destes casos.

\section{Agradecimentos}

Agradecemos aos nossos alunos, fonte contínua de inspiração e incentivo para a melhoria do ensino na administração. Um agradecimento especial ao prof. Herbert Kimura que enquanto editor da RAC nos incentivou a construir este manuscrito, extensivo a todos os colegas professores que enfrentam em seu cotidiano os nossos mesmos desafios.

\section{Nota}

\footnotetext{
${ }^{1}$ Este artigo é oriundo de convite realizado pelo então Editor-chefe da RAC, Prof. Herbert Kimura. A primeira autora é a exeditora associada da seção de Casos para Ensino, e o coautor é membro do Conselho Editorial da RAC.
}

\section{Contribuições}

$1^{\circ}$ autor: Reflexões sobre o método e a estrutura base para um bom caso para ensino, construção da revisão teórica de suporte e revisão final do manuscrito.

$2^{\circ}$ autor: Reflexões sobre o método e a estrutura base para um bom caso para ensino, construção da revisão teórica de suporte e revisão final do manuscrito.

\section{Referências}

Araújo, M. S. M., \& Sousa, S. C. T. (2016). Casos para o ensino: Uma abordagem a partir da linguística de texto. Métodos e Pesquisa em Administração, 1(2), 69-79. Recuperado de http://www.periodicos.ufpb.br/ojs/index.php/mepad/article/view/32193/16726

Associação Nacional de Pós-Graduação e Pesquisa em Administração. (2007b, novembro). Encontro de Ensino e Pesquisa em Administração e Contabilidade, Recife, PE, Brasil, 1. Recuperado de http://www.anpad.org.br/ anpad/eventos.php?cod_evento=18\&cod_evento_edicao=35

Associação Nacional de Pós-Graduação e Pesquisa em Administração. (2007a, setembro). Encontro Nacional da Associação Nacional de Pós-Graduação e Pesquisa em Administração, Rio de 
Janeiro,

RJ, Brasil,

31.

Recuperado

de

http://www.anpad.org.br/ anpad/eventos.php?cod_evento=1\&cod_evento_edicao=33

Associação Nacional de Pós-Graduação e Pesquisa em Administração. (2008, setembro). Encontro Nacional da Associação Nacional de Pós-Graduação e Pesquisa em Administração, Rio de Janeiro, RJ, Brasil, $32 . \quad$ Recuperado de http://www.anpad.org.br/ anpad/eventos.php?cod_evento=1\&cod_evento_edicao=38

Associação Nacional de Pós-Graduação e Pesquisa em Administração. (2009b, novembro). Encontro de Ensino e Pesquisa em Administração e Contabilidade, Curitiba, PR, Brasil, 2. Recuperado de http://www.anpad.org.br/ anpad/eventos.php?cod_evento=18\&cod_evento_edicao=47

Associação Nacional de Pós-Graduação e Pesquisa em Administração. (2009, setembro). Encontro Nacional da Associação Nacional de Pós-Graduação e Pesquisa em Administração, São Paulo, SP, Brasil, $33 . \quad$ Recuperado de http://www.anpad.org.br/ anpad/eventos.php?cod_evento=1\&cod_evento_edicao=45

Associação Nacional de Pós-Graduação e Pesquisa em Administração. (2010, setembro). Encontro Nacional da Associação Nacional de Pós-Graduação e Pesquisa em Administração, Rio de Janeiro, RJ, Brasil, $34 . \quad$ Recuperado de http://www.anpad.org.br/ anpad/eventos.php?cod_evento=1\&cod_evento_edicao=53

Associação Nacional de Pós-Graduação e Pesquisa em Administração. (2011b, novembro). Encontro de Ensino e Pesquisa em Administração e Contabilidade, João Pessoa, PB, Brasil, 3. Recuperado de http://www.anpad.org.br/ anpad/eventos.php?cod_evento=18\&cod_evento_edicao=60

Associação Nacional de Pós-Graduação e Pesquisa em Administração. (2011a, setembro). Encontro Nacional da Associação Nacional de Pós-Graduação e Pesquisa em Administração, Rio de Janeiro, RJ, Brasil, $35 . \quad$ Recuperado de http://www.anpad.org.br/ anpad/eventos.php?cod_evento=1\&cod_evento_edicao $=58$

Associação Nacional de Pós-Graduação e Pesquisa em Administração. (2012, setembro). Encontro Nacional da Associação Nacional de Pós-Graduação e Pesquisa em Administração, Rio de Janeiro, RJ, Brasil, $36 . \quad$ Recuperado de http://www.anpad.org.br/ anpad/eventos.php?cod_evento=1\&cod_evento_edicao=63

Associação Nacional de Pós-Graduação e Pesquisa em Administração. (2013b, novembro). Encontro de Ensino e Pesquisa em Administração e Contabilidade, Brasília, DF, Brasil, 4. Recuperado de http://www.anpad.org.br/ anpad/eventos.php?cod_evento=18\&cod_evento_edicao=70

Associação Nacional de Pós-Graduação e Pesquisa em Administração. (2013a, setembro). Encontro Nacional da Associação Nacional de Pós-Graduação e Pesquisa em Administração, Rio de Janeiro, RJ, Brasil, $37 . \quad$ Recuperado de http://www.anpad.org.br/ anpad/eventos.php?cod_evento=1\&cod_evento_edicao=68

Associação Nacional de Pós-Graduação e Pesquisa em Administração. (2014, setembro). Encontro Nacional da Associação Nacional de Pós-Graduação e Pesquisa em Administração, Rio de Janeiro, RJ, Brasil, $38 . \quad$ Recuperado de http://www.anpad.org.br/ anpad/eventos.php?cod_evento=1\&cod_evento_edicao=73

Associação Nacional de Pós-Graduação e Pesquisa em Administração. (2015b, novembro). Encontro de Ensino e Pesquisa em Administração e Contabilidade, Salvador, BA, Brasil, 5. Recuperado de http://www.anpad.org.br/ anpad/eventos.php?cod_evento=18\&cod_evento_edicao=79

Associação Nacional de Pós-Graduação e Pesquisa em Administração. (2015a, setembro). Encontro Nacional da Associação Nacional de Pós-Graduação e Pesquisa em Administração, Belo 
Horizonte, MG, $\quad$ Brasil, $39 . \quad$ Recuperado de http://www.anpad.org.br/ anpad/eventos.php?cod_evento=1\&cod_evento_edicao=78

Associação Nacional de Pós-Graduação e Pesquisa em Administração. (2016, setembro). Encontro Nacional da Associação Nacional de Pós-Graduação e Pesquisa em Administração, Costa do Sauípe, BA, Brasil, $40 . \quad$ Recuperado de http://www.anpad.org.br/ anpad/eventos.php?cod_evento=1\&cod_evento_edicao=83

Associação Nacional de Pós-Graduação e Pesquisa em Administração. (2017, outubro). Encontro Nacional da Associação Nacional de Pós-Graduação e Pesquisa em Administração, São Paulo, SP, Brasil, $41 . \quad$ Recuperado de http://www.anpad.org.br/ anpad/eventos.php?cod_evento=1\&cod_evento_edicao=89

Associação Nacional de Pós-Graduação e Pesquisa em Administração. (2018b, maio). Encontro de Ensino e Pesquisa em Administração e Contabilidade, Porto Alegre, RS, Brasil, 6. Recuperado de http://www.anpad.org.br/ anpad/eventos.php?cod_evento=18\&cod_evento_edicao=90

Associação Nacional de Pós-Graduação e Pesquisa em Administração. (2018a, outubro). Encontro Nacional da Associação Nacional de Pós-Graduação e Pesquisa em Administração, Curitiba, PR, Brasil, $42 . \quad$ Recuperado de http://www.anpad.org.br/ anpad/eventos.php?cod_evento=1\&cod_evento_edicao=93

Bangs, J. (2011). Experiential learning in an organizational leadership program. Journal of College Teaching and Learning, 8(10), 29-34. https://doi.org/10.19030/tlc.v8i10.6109

Barnes, L. B., Chistensen, C. R., \& Hansen, A. J. (1994). Teaching and the case method: Text, cases, and readings ( $3 \mathrm{a}$ ed.). Boston: Harvard Business School Press.

Bonoma, T. (1989). Learning with cases. Boston: Harvard Business School Publishing.

Booth, C., Bowie, S., Jordan, J., \& Rippin, A. (2000). The use of the case method in large and diverse undergraduate business programmes: Problems and issues. International Journal of Management Education, $\quad 1, \quad 62-75 . \quad$ Retrieved from https://www.researchgate.net/profile/Ann_Rippin/publication/253150414_The_Use_of_the_Cas e_Method_in_Large_and_Diverse_Undergraduate_Business_Programmes_Problems_and_Issue s/links/5449f9230cf2f6388084d892/The-Use-of-the-Case-Method-in-Large-and-DiverseUndergraduate-Business-Programmes-Problems-and-Issues.pdf. https://doi.org/10.3794/ijme.11.f

Chang, J. (2003). Strategic management: An evaluation of the use of three learning methods in Hong Kong. Developments in Business Simulation \& Experiential Learning, 30, 146-151. Retrieved from http://citeseerx.ist.psu.edu/viewdoc/download?doi=10.1.1.522.9701\&rep=rep1\&type=pdf

Cho, Y., Egan, T. M. (2010). The state of the art of action learning research. Advances in Developing Human Resources, 12(2), 163-180. https://doi.org/10.1177/1523422310367881

Ellet, W. (2007). The case study handbook: How to read, discuss, and write persuasively about cases. Boston: Harvard Business School Press.

Ellet, W. (2008). Manual de estudo de caso: Como ler, discutir e escrever casos de forma persuasiva. Porto Alegre: Bookman.

Erskine, J. A., Leenders, M. R., \& Mauffette-Leenders, L.A. (1998). Teaching with cases. London, Ontario, CA: Richard Ivey School of Business.

Faria, M. D., \& Figueiredo, K.F. (2013). Casos de ensino no Brasil: Análise bibliométrica e orientações para autores. Revista de Administração Contemporânea, 17(3), 176-197. Recuperado de http://www.scielo.br/pdf/rac/v17n2/04.pdf. http://doi.org/10.1590/S1415-65552013000200004

RAC, Rio de Janeiro, v. 22, n. 5, art. 6, pp. 745-761, setembro/outubro, 2018, www.rac.anpad.org.br 
Harling, K., \& Akridge, J. (1998). Using the case method of teaching. Agribusiness, 14(1), 1-14. https://doi.org/10.1002/(SICI)1520-6297(199801/02)14:1<1::AID-AGR1>3.0.CO;2-8

Hawes, J. M. (2004). Teaching is not telling: The case method as a form of interactive learning. Journal for Advancement of Marketing Education, 5, 47-54. Retrieved from http://www.mmaglobal.org/publications/JAME/JAME-Issues/JAME-2004-Vol05Issue1/JAME-2004-Vol05-Issue1-Hawes-pp47-54.pdf

Herbert, A., \& Stenfors, S. (2007). Chosing experiential learning methods for management education: The fit of action learning and problem-based-learning. In M. Reynolds \& R. Vince (Eds.), Experiential learning and management education (pp. 221-238). New York: Oxford University Press.

Ikeda, A. A., Veludo-de-Oliveira, T. M., \& Campomar, A. A. (2006). O caso como estratégia de ensino na área de Administração. Revista de Administração, 41(2), 147-157. Recuperado de https://gvpesquisa.fgv.br/sites/gvpesquisa.fgv.br/files/arquivos/veludo__o_caso_como_estrategia_de_ensino_na_area_de_administracao.pdf

Jarvis, P. (2006). Towards a comprehensive theory of human learning: Lifelong learning and the learning society. New York: Routledge.

Jennings, D. R. (1996). Strategic management and the case method. Journal of Management Development, 15(9), 4-12. https://doi.org/10.1108/02621719610146211

Jennings, D. (2002). Strategic management: An evaluation of the use of three learning methods. The Journal of Management Development, 21(9/10), 655-665. https://doi.org/10.1108/02621710210441658

Kolb, A. Y., Kolb, D. A. (2005). Learning styles and learning spaces: Enhancing experiential learning in higher education. Academy of Management Learning \& Education, 4(2), 193-212. https://doi.org/10.5465/amle.2005.17268566

Kolb, D. (1984). Experiential learning: Experience as the source of learning and development. New Jersey: Prentice Hall.

Leenders, M. R., Mauffette-Leenders, L. A., \& Erskine, J. A. (2001). Writing cases (4th ed.). London, Ontario, CA: Richard Ivey School of Business.

Machado, A. G. C., \& Callado, A. A. C. (2008). Precauções na adoção do método de estudo de caso para o ensino de administração em uma perspectiva epistemológica [Edição Especial]. Cadernos EBAPE.BR, 6, 1-10. http://dx.doi.org/10.1590/S1679-39512008000500006

Mauffette-Leenders, L. A., Erskine, J. A., \& Leenders, M. R. (1997). Learning with cases. London, Ontario, CA: Richard Ivey School of Business.

Merriam, S. B., \& Bierema, L. L. (2014). Adult learning: Linking theory and practice. San Francisco: Jossey-Bass.

Moon, J. A. (2004). A handbook of reflective and experiential learning: Theory and practice. London: Routledge Falmer.

Revans, R. (2011). ABC of action learning. Burlington, USA: Gower.

Roberts, M. (1999). Developing a teaching case [Teaching materials, 9-900-001]. Boston: Harvard Business School Publishing.

Rocha, A. da, \& Mello, R. C. de (2000). Marketing de serviços: Casos brasileiros. São Paulo: Atlas. 
Roesch, S. M. A. (2007). Casos de ensino em administração: Notas sobre a construção de casos para ensino. Revista de Administração Contemporânea, 11(2), 213-234. http://dx.doi.org/10.1590/S1415-65552007000200012

Roesch, S. M. A., \& Fernandes, F. (2007). Como escrever casos para o ensino de administração. São Paulo: Atlas.

Rollag, K. (2010). Teaching business cases online through discussion boards: Strategies and best practices. Journal of Management Education, 34(4), 499-526. https://doi.org/10.1177/1052562910368940

Rosier, G. (2002). Using reflective reports to improve the case method. The Journal of Management Development, 21(7/8), 589-597. https://doi.org/10.1108/02621710210437563

Silva, A. B. (2014, setembro). Reflexões teórico-práticas de um sistema de aprendizagem-em-ação para a educação em administração. Anais do Encontro Nacional da Associação Nacional de PósGraduação e Pesquisa em Administração, 1, Rio de Janeiro, RJ, 28.

Silva, A. B. (2016). Action learning: Lecturers, learners, and managers at the center of management education. In M. T. Lepeley, E. V. Kimakovitz, \& R. Bardy (Eds.), Human centered management in executive education: Global imperatives, innovation and new directions (pp. 126-139). London: Palgrave Macmillan.

Silva, A. B., Lima, T. B., Sonaglio, A. L. B., \& Godoi, C. K. (2012). Dimensões de um sistema de aprendizagem em ação para o ensino de administração. Administração: Ensino e Pesquisa, 13(1), 9-41. https://doi.org/10.13058/raep.2012.v13n1.97

Smith, P. A. C. (2001). Action learning and reflective practice in project environments that are related to leadership development. Management Learning, 32(1), 31-48. https://doi.org/10.1177/1350507601321003

Sofo, F., Yeo, R. K., \& Villafañe, J. (2010). Optimizing the learning in action learning: Reflective questions, levels of learning and coaching. Advances in Developing Human Resources, 12(2), 205-224. https://doi.org/10.1177/1523422310367883

\title{
Dados dos Autores
}

\author{
Anete Alberton \\ Rua João Coan, 400, 88161-064, Biguaçu, SC, Brasil. \\ E-mail: anete@univali.br \\ Anielson Barbosa da Silva \\ Cidade Universitária, s/n - Castelo Branco III, 58051-900, João Pessoa, PB, Brasil. \\ E-mail: anielson@uol.com.br
}

